\title{
Differences in Postural Oscillation during Quiet Stance Alone and Quiet Stance Following Sit-To-Stand Movement in Children with Cerebral Palsy
}

\author{
Pavão SL*, de Oliveira Sato T and Rocha NACF \\ Department of Physiotherapy, Neuropediatrics Section, \\ Federal University of São Carlos, Rod. Washington Luis, \\ km 235, 13565-905, São Carlos-SP, Brazil \\ *Corresponding author: Sílvia Leticia Pavão, \\ Department of Physiotherapy, Neuropediatrics Section, \\ Federal University of São Carlos, Rod. Washington Luis, \\ km 235, 13565-905, São Carlos-SP, Brazil
}

Received: August 23, 2017; Accepted: September 18, 2017; Published: September 25, 2017

\begin{abstract}
We evaluated differences in CoP trajectories during quiet stance alone (QSA) and quiet stance following sit to stand (STS) (QSFS) in typical children (TD) and children with cerebral palsy (CP). Forth two TD, 23 spastic CP were evaluated. The assessment during QSA occurred with the maintenance on a force plate during 30 seconds. For evaluation in QSFS children were instructed to rising from a bench and maintain stance for 30 . We observed higher oscillation (AP and ML Amplitude of CoP displacement, Velocity and Area of CoP oscillation) in QSFS than in QSA. All the variables, except AP Amp, presented higher values for the CP group compared with TD. With exception of the variable AP Amp, all the other ones presented interaction between condition and group. CP group present higher CoP oscillation than TD, these values were higher in QSFS.
\end{abstract}

Keywords: Children; Cerebral palsy; Sit-to-stance movement; Postural control; Dynamical activity

\section{Introduction}

Many of the activities performed by children in daily routine demand the maintenance of quiet stance in order to reach adaptive success [1]. Some examples include: the waiting time in lines, activities involving washing hands and brushing teeth, as well as social activities performed during stance [2]. The maintenance of standing posture involves the ability to keep the center of pressure $(\mathrm{CoP})$ within the limits of the support base and suitable alignment between body segments [3-5] to keep balance and avoid falls [6,7].

A literature review reveals a large amount of studies addressing postural control during quiet stance in children with cerebral palsy (CP) [8-13]. According to these studies children with mild motor impairments present reduced lautomaticity in postural sway [12] and less adaptive patterns of postural control regulation in this posture $[3,8]$ than typical children. Nashner et al. [5] report an inverted pattern of muscle activation in lower limbs (distal to proximal) to keep balance during quiet stance. In addition, the crouch posture often observed in these children contributes to the greater postural sway that they present.

However, the experimental design of the existing studies is not able to reproduce functional experienced circumstances in daily life. In most of the studies children were instructed to keep standing posture as static as possible by a limited period of time [13], and in some cases they had to perform a concurrent cognitive task $[12,14,15]$ or sensory manipulations $[8,11]$ were required while remaining static.

Nevertheless, daily routine tasks involving upright stance maintenance commonly occur following functional movements, such as postural transitions or body displacements. Sit-to-stand (STS) movement is an example of a task that commonly precedes stance maintenance. The sequence of muscle activation and the positioning of the center of mass (CoM) occurring during the body movement of rising from a chair modifies postural demands to keep stability $[4,7]$. STS movement requires specific postural adjustments, such as, muscle co-activation in lower limbs to decelerate CoM movement $[16,17]$, interjoint coordination in ankle, knee and hip joints to keep body alignment and a stable base of support [18]. These adjustments are believed to influence balance in the following quiet stance maintenance.

In fact, when considering a sequence of motor tasks, the preceding task exerts biomechanical influences on the following, since muscle and joint receptors, as well as other sensory system organs, catch the information coming from the movement in order to guide and correct the performed action [19]. In addition, the body movement changes the alignment between the segments creating specific demands for postural control system [5]. In this context, the task preceding upright stance maintenance seems to challenge postural control in a different way than the upright stance maintenance alone [20].

Studies have addressed postural control in CP children during dynamical circumstances such as, STS movement [16,21,22], gait $[23,24]$, gait initiation [6] and gait initiation following STS movement [25]. These studies report a greater postural sway observed in CP children, as well as a greater ankle excursion in frontal and transverse planes during the movement [26], possibly due to the lack of coordination of ankle muscles activation reported in CP [27].

Nevertheless, none of the above studies have addressed postural control during quiet stance preceded by some of these functional body movements that are so common in daily living. This lack of studies is even more significant when considering the population of children with CP. Addressing this gap would allow us to understand how body movement and alignment might influence balance maintenance, guiding rehabilitation professionals in the selection of the more
Phys Med Rehabil Int - Volume 4 Issue 3 - 2017 ISSN : 2471-0377 | www.austinpublishing group.com Pavão et al. (C) All rights are reserved
Citation: Pavão SL, de Oliveira Sato T and Rocha NACF. Differences in Postural Oscillation during Quiet Stance Alone and Quiet Stance Following Sit-To-Stand Movement in Children with Cerebral Palsy. Phys Med Rehabil Int. 2017; 4(3): 1121. 
challenging activities to improve balance and stability.

Thus, the aim of our study was to evaluate differences in patterns of postural oscillation during quiet stance alone and quiet stance following STS movement. For this purpose we evaluated children with cerebral palsy and children with typical development.

We believe that postural demands in upright stance position following STS movement are greater than the ones of keeping stance alone. In this context we expected to find greater postural oscillation in the former conditions than in the later one. Moreover, despite children with mild CP were able to perform STS movement independently and to keep stance following this postural transition we believe that in stance maintenance following STS movement children with $\mathrm{CP}$ will present greater postural oscillations compared with children with typical development.

\section{Methods \\ Participants}

We evaluated three groups of participants. The typicallydeveloping group (TD) included 42 healthy children, 19 male and 23 female, with ages from 5 to 15 years (mean \pm SD; Age: $10 \pm 2.9$ years; Height: $143 \pm 19.1 \mathrm{~cm}$; Weight: $41.5 \pm 17.2 \mathrm{Kg}$ ). Participants with current lower limb injuries, or who had any cardiovascular, pulmonary, neurological, or systemic conditions that limited physical activity were excluded from the study.

The other group included 23 children with spastic CP , 15 male and 8 female, with ages from 5 to 15 years (mean \pm SD; Age: 9.8 \pm 3.3 years; Height: $129.7 \pm 33.4 \mathrm{~cm}$; Weight: $31.3 \pm 12.4 \mathrm{Kg}$ ). The participants were classified by Gross Motor Function Classification System (GMFCS) as level I (15 children) or level II (8 children). For statistical analyses the CP group was divided according to the level of GMFCS.

The inclusion criteria for the CP group were as follows: (a) ability to follow simple commands; (b) ability to come to a standing position independently. Exclusion criteria were: (a) loss of passive joint mobility in trunk or lower limbs, which may indicate presence of deformities and contractures; (b) orthopedic surgery and/ or botulinum toxin injection in the previous 12 and 6 months, respectively; (c) any muscle tone impairment other than spasticity, such as ataxia, dystonia, and/or hypotonia.

The local Ethics Committee for Human Research approved the study, which is in agreement with the Declaration of Helsinki and the resolution 196/96 from National Health Council. Children were admitted in the study following informed written parental consent. Children were recruited in rehabilitation centers specialized in child care and in regular schools.

\section{Procedures}

The anthropometric data of all the children were previously collected.

For the assessment of postural control during quiet stance alone, children were asked to assume upright standing posture standing on a force platform (Bertec400) with their feet parallel to and aligned with the side of their feet and to remain standing for 30 seconds. Following this preceding time, we initiated our data collection instructing them to remain on the force plate as quietly as possible for 30 seconds while looking ahead at a fixed point located one meter away [28]. Each child performed a total of three trials.

For postural control testing during quiet stance following STS movement children were initially seated in a bench without trunk or upper limbs support. Their hips, knees and ankles were flexed at $90^{\circ}$ and their feet were positioned on a force plate, at an acquisition rate of $100 \mathrm{~Hz}$. The participants were tested in barefoot. After a verbal command, the children were asked to assume upright standing position in a self-selected speed, keeping their arms folded across the chest when they stood (to prevent them from using their upper limbs to push up of the bench). Immediately after standing up, they were instructed to remain in static stand up for 30 seconds. The task was performed for a total of three times.

A circle was drawn at the center of the platform to orient feet placement before each trial in both conditions, which provided consistency of the initial position across trials. There was no discrepancy of the support base adopted by the children among the performed conditions. Children did not perform training tasks in none of the conditions.

For analysis of body oscillations, in both conditions, we used the mean of the three performed trials.

\section{Data analysis}

Data from the force platform were processed and filtered ( $4^{\text {th }}$ order Butterworth filter, with a low pass frequency of $5 \mathrm{~Hz}$ ) on Matlab (Mathworks Inc., Natick, MA, USA); outcome measures were computed on the same software. Data were normalized by the participants' body weight.

STS movement can be divided into three different phases $[16,22]$. The criteria for STS movement division into phases were: preparation phase $(\mathrm{F} 1)$ the beginning was determined by a decrease in vertical force greater than $2.5 \%$ relative to the weight of the feet on the platform, and the end was determined by the vertical peak force; rising phase (F2), measurement began with the vertical peak force on the platform and ended when the vertical force matched the body weight; stabilization phase (F3) was determined by the point at which the vertical force reached the body weight, and the end was determined by a vertical force oscillation of approximately $2.5 \%$ of the body weight [29]. The phase division of STS movement is shown in Figure 1.

For postural oscillation analyses during quiet stance following STS movement, in each trial, we cut of the trial as soon as F3 was reached and conducted the data analysis in the remaining 30 seconds of force plate capture.

The following variables of postural oscillation were analyzed in each of the conditions: (1) anterior-posterior (AP Amp) and mediallateral (ML Amp) amplitude of CoP displacement: Correspond to the variance of the $\mathrm{CoP}$ values between the maximum and minimum displacement in these directions. Greater values of these variables indicate balance difficulties; (2) Area of CoP oscillation (Area): Corresponds to the dispersion of the oscillation considering the AP and ML directions. This variable estimates the dispersion of CoP data by the calculation of the statokinesiogram area. Area was calculated by the main components analysis, which calculates an ellipse comprising 


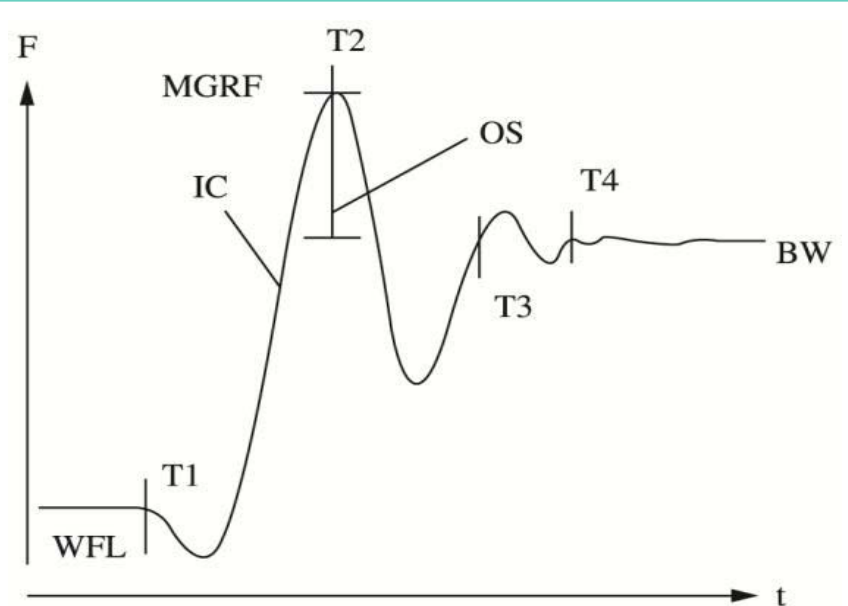

Figure 1: Schematic representation of the division process of the three different phases of the sit-to-stand movement. Preparation phase (T1-T2); rising phase (T2-T3); and stabilization phase (T3-T4). BW, body weight; MGRF (maximum ground reaction force); OS (overshoot); IC (incline) WFL (weight of feet/legs at rest); T1 (start of movement); T2 (seat-off); T3 (extension of body); T4 (end of movement). Source: Kralj et al.

95\% of the CoP data. The two axes of this ellipse are calculated from the measures of dispersion of CoP signals. The higher their values, the greater the balance deficits; (3) mean velocity (Vel) of CoP oscillation: Reflects how fast the body oscillation occurred. Its calculation used the following formula: $d=\sqrt{\left(x_{2}-x_{1}\right)^{2}+\left(y_{2}-y_{1}\right)^{2}}$. The higher the velocity of $\mathrm{CoP}$ oscillation, the greater the difficulties to keep balance [4].

\section{Statistical analysis}

Descriptive values (means, standard deviations) across trials were first obtained. Data distribution was tested for normality and homoscedasticity, using the Kolmogorov-Smirnov and Levene tests, respectively. The tests revealed a normal distribution of the data.

A two-way repeated measures MANOVA (Multivariate Analysis Of Variance) was run to verify the effects of postural condition (quiet stance alone and quiet stance following STS) and group (typical, CP GMFCS I and CP GMFCS II) across postural oscillation variables (AP Amp, ML Amp, Vel and Area). Tukey's post-hoc was used for multiple comparisons.

\section{Results}

We observed a main effect of postural oscillation in the performed conditions of quiet stance $\left(\mathrm{F}_{(5.58)}=82.8, \mathrm{p}<0.01\right)$. We also observed within subjects effects of interaction between condition and group $\left(\mathrm{F}_{(10,118)}=6,5, \mathrm{p}<0.01\right)$. In addition, between subjects effects of group were observed $\left(\mathrm{F}_{(10,118)}=7,5, \mathrm{p}<0.01\right)$. The values of the mean and interactions effects of each analyzed variable are shown in Table 1.

There was a significant interaction between condition and group on the combined dependent variables, $\mathrm{F}_{(10,116)}=6.914, \mathrm{p}<0.01$, Wilks' $\Lambda=0.393$, partial $\eta 2=0.373$. Follow up univariate two-way ANOVAs were run and showed a significant interaction effect between condition and group for ML Amp $\left(\mathrm{F}_{(2,62)}=12.582, \mathrm{p}<0.01\right.$, partial $\eta 2=0.289)$, Vel $\left(\mathrm{F}_{(2,62)}=10.246, \mathrm{p}<0.01\right.$, partial $\left.\eta 2=0.248\right)$ and Area $\left(\mathrm{F}_{(2,62)}=11.434, \mathrm{p}<0.01\right.$, partial $\left.\eta 2=0.269\right)$, but not for $\operatorname{AP} \operatorname{Amp}\left(\mathrm{F}_{(2,62)}\right.$
Table 1: Main and interaction effects of group (between-subjectfactor, three levels: Typical Development, Cerebral Palsy GMFCS I and Cerebral Palsy GMFCS II) and condition (within-subject factor, two levels: orthostatic maintenance by itself and orthostatic maintenance following STS ) on AnteroPosterior Amplitude (AP Amp), Medio-Lateral Amplitude (ML Amp), Velocity (Vel) and Area of CoP oscillation.

\begin{tabular}{|c|c|c|c|c|c|c|}
\hline Variable & \multicolumn{3}{|c|}{ Condition } & \multicolumn{3}{c|}{ Condition X Group } \\
\hline & V & F & P-value & V & F & P-value \\
\hline AP Amp & 0.8 & $(1,62)=261,3$ & $P<0.01$ & 0.3 & $(2,62)=0.971$ & 0.384 \\
\hline ML Amp & 0.6 & $(1,62)=134,1$ & $P<0.01$ & 0.2 & $(2,62)=12.5$ & $P<0.01$ \\
\hline Vel & 0.8 & $(1,62)=290.9$ & $P<0.01$ & 0.2 & $(2,62)=10.2$ & $P<0.01$ \\
\hline Area & 0.7 & $(1,62)=173.8$ & $\mathrm{P}<0.01$ & 0.2 & $(2,62)=11.4$ & $P<0.01$ \\
\hline
\end{tabular}

$=0.971, \mathrm{p}=0.384$, partial $\eta 2=0.030)$.

The means, standard error and confidence interval of the variables for each of the groups in each of the conditions are shown in Table 2.

A simple main effects analysis was conducted for ML Amp, Vel and Area. There was a statistically significant difference between conditions for all groups for the variables Amp ML, Vel and Area, with higher values observed in the condition of quiet standing following STS. There was also a statistically significant difference between typical children and children with CP, both level I and II (which did not present differences) in both conditions for the variables Amp ML, Area and Vel. Children with CP showed higher values in these variables than typical ones.

For AP Amp there was no statistically significant difference between groups $\left(\mathrm{F}_{(2,62)}=1.158, \mathrm{p}=0.321\right.$, partial $\left.\eta 2=0.036\right)$. The difference between conditions was significant $\left(\mathrm{F}_{(1,62)}=261.313, \mathrm{p}<\right.$ 0.001 , partial $\eta 2=0.808$ ), with higher values observed in the condition of quiet standing following STS.

\section{Discussion}

Our study aimed to investigate differences in patterns of postural oscillation in quiet stance alone and quiet stance following STS movement. According to the results all the groups of children presented a larger amount of postural oscillation for all the analyzed variables in the condition of quiet stance following STS movement than in quiet stance alone. Moreover, with the exception of AP Amp of CoP displacement, that did not present differences between the three groups, all the other variables showed group differences across conditions.

We have no knowledge of studies addressing patterns of postural oscillation in quiet stance following STS movement neither in children with CP nor in other populations. Buckley et al. [25] evaluated postural control during sit-to-walk transitions in individuals with Parkinson disease. Nevertheless, although the authors have considered a functional activity following STS movement (gait initiation) they did not compare the results obtained with a control condition (only STS movement, or only gait initiation, for example). Even so, the authors verified that the limitations in proprioception, movement speed, muscular strength, and reduced general mobility observed in the patients with Parkinson disease, determined greater time to complete the ascending phase of STS, as well as slower velocity leading to shorter steps compared with the control group. 
Table 2: Values of means, Standard errors and confidence interval for all the analyzed variables in each of the groups and condition evaluated.

\begin{tabular}{|c|c|c|c|c|c|c|}
\hline \multirow{2}{*}{ Variable } & \multirow{2}{*}{ Group } & \multirow{2}{*}{ Condition } & \multirow{2}{*}{ Mean } & \multirow{2}{*}{ StanceardError } & \multicolumn{2}{|c|}{ 95\% Confidencelnterval } \\
\hline & & & & & LowerBound & UpperBound \\
\hline \multirow{6}{*}{$\begin{array}{l}\text { AP Amp } \\
(\mathrm{cm})\end{array}$} & \multirow{2}{*}{ CP I } & QuietStance & 3.1 & 0.2 & 2.6 & 3.6 \\
\hline & & QuietStancepost STS & 7.6 & 0.4 & 6.7 & 8.4 \\
\hline & \multirow{2}{*}{ CP II } & QuietStance & 3.6 & 0.3 & 2.9 & 4.2 \\
\hline & & QuietStance post STS & 7.7 & 0.5 & 6.5 & 8.9 \\
\hline & \multirow{2}{*}{ Typical } & QuietStance & 2.6 & 0.1 & 2.3 & 2.9 \\
\hline & & QuietStance post STS & 7.6 & 0.2 & 7.1 & 8.1 \\
\hline \multirow{6}{*}{$\begin{array}{l}\text { ML Amp } \\
\quad(\mathrm{cm})\end{array}$} & \multirow{2}{*}{ CP I } & QuietStance & 3.6 & 0.2 & 3.1 & 4.1 \\
\hline & & QuietStance post STS & 10.8 & 0.6 & 9.5 & 12.1 \\
\hline & \multirow{2}{*}{ CP II } & QuietStance & 4.5 & 0.3 & 3.9 & 5.2 \\
\hline & & QuietStance post STS & 8.06 & 0.9 & 6.2 & 9.8 \\
\hline & \multirow{2}{*}{ Typical } & QuietStance & 2.5 & 0.1 & 2.2 & 2.7 \\
\hline & & QuietStance post STS & 5.8 & 0.3 & 5.03 & 6.6 \\
\hline \multirow{6}{*}{$\begin{array}{l}\text { Velocity } \\
(\mathrm{cm} / \mathrm{s})\end{array}$} & \multirow{2}{*}{ CP I } & QuietStance & 1.8 & 0.1 & 1.6 & 2.09 \\
\hline & & QuietStance post STS & 3.4 & 0.1 & 3.1 & 3.8 \\
\hline & \multirow{2}{*}{ CP II } & QuietStance & 1.9 & 0.1 & 1.6 & 2.3 \\
\hline & & QuietStance post STS & 3.1 & 02 & 2.6 & 3.6 \\
\hline & \multirow{2}{*}{ Typical } & QuietStance & 1.4 & 0.7 & 1.2 & 1.5 \\
\hline & & QuietStance post STS & 2.3 & 0.1 & 2.1 & 2.5 \\
\hline \multirow{4}{*}{$\begin{array}{l}\text { Area } \\
\left(\mathrm{cm}^{2}\right)\end{array}$} & \multirow{2}{*}{ CP I } & QuietStance & 4.6 & 0.5 & 3.5 & 5.7 \\
\hline & & QuietStance post STS & 15.7 & 1.2 & 13.3 & 18.1 \\
\hline & CP II & QuietStance & 7.4 & 0.7 & 5.9 & 9 \\
\hline & Typical & QuietStance post STS & 7.9 & 0.7 & 6.4 & 9.3 \\
\hline
\end{tabular}

The main point of our study is that the preceding motor task exerts influence on balance maintenance. This is especially important taking into account that balance maintenance during standing posture is almost all the time proceeded by body movements, such as STS movement. The postural demands observed in quiet stance following STS movement are higher than the ones involved with the maintenance of the body in static stand posture alone, since the former condition involves the change of the body system from a movement condition to a stationary state. This change requires deceleration of the CoM to break the body motion and keep stability during standing posture. Movement deceleration requires greater levels of concentriceccentric muscle control of the lower limbs for proper interjoint coordination and muscular co-contraction to keep alignment between body segments and to regulate CoM positioning within the limits of support base [17,30], as well as refined neuromotor balance strategies [31] such as hip and ankle strategies of stability [5]. These previous postural adjustments influence and challenge the following balance maintenance, placing greater difficulties to the task [19].

Previous studies have reported the high biomechanical demand involved with rising from a chair $[16,20,21,32]$. Among the requirements are the performance of the movement against the gravity and a reduction in the base of support, demanding considerable muscular strength, knee extensor torque and postural strategies to keep the CoP within the limits of the base of support $[24,26]$. Moreover, the last phase of STS movement (stabilization phase - F3) involves movement deceleration, which seems to create an additional biomechanical demand [17] and greater postural instability [16,20]. Therefore, the STS movement as a preceding motor task seems to influence the following balance maintenance in static posture, further challenging the postural control system.

Despite the reduced stability we observed in children with $\mathrm{CP}$ during the performed tasks, the mild motor impairment of the participants in this study did not hinder the accomplishment of stance maintenance after rising up from a bench. In fact, children with $\mathrm{CP}$, due to their muscular and proprioceptive impairments in ankle joint $[17,33]$, present increased trunk excursion during STS movement compared with typical children [32]. However, the end of the task (i.e. the braking of the movement) seems to lack stability due to the neuromuscular deficits these children present.

Moreover, the differences in CoP sway observed among the two conditions could be analyzed in the light of the effects of sensory information in stance following STS movement. STS transition involves body movement in space and changes in head position [32], which affect the vestibular system [19]. This transition also 
challenges postural control because of the high anterior-posterior and vertical velocities of movement and the large forward trunk flexion that it involves [34]. These factors provide stimulation in vestibular receptors [35], which may modify the coordination of motor responses [19] challenging postural stability when stance posture is attained. Based on these statements, stance maintenance following STS movement can be expected to involve greater CoP displacement and velocity of oscillation compared with stance maintenance alone in order to explore the base of support, capturing additional sensory information [17]. These increases observed in all analyzed variables in the condition following STS movement might be consequences of adopted postural strategies (ankle and hip strategies) which ensure stability and avoid falls [5].

As could also be expected, the increases observed in CoP trajectories following STS movement (ML Amp, Vel and Area) were greater in children with CP than in TD children. In fact, when considering simple task sequences, such as the ones addressed by our study (STS $\rightarrow$ quiet stance), besides the fact that the preceding motor task influences the following one, the motor system uses sensory information coming from the movement to provide feedback in order to control and correct potential movement errors [19]. Nevertheless, children with CP present important deficits in sensory integration $[16,36,37]$, which can result in poorer balance control than typical children.

Moreover, the maintenance of quiet stance following STS movement demands anticipatory and compensatory postural adjustments to keep stability after a dynamic activity [38]. Anticipatory postural adjustments are required in the beginning of the movement in order to counteract the expected mechanical effects of perturbation in a feed forward manner [10]. Compensatory postural adjustments occur in the end of the movement reducing the effects of postural perturbations during the movement [9]. Nevertheless, children with CP with GMFCS levels I and II present high co-activation in lower limb muscles [1,5], as well as decreasing anticipatory and compensatory postural adjustments $[9,10]$ which may have contributed to the greater postural oscillation during quiet stance following STS movement in this study.

We did not find any differences between groups for the variable AP Amp of CoP displacement. The muscle deficits in antigravity musculature [10] as well as mechanical changes in posture observed in children with $\mathrm{CP}$, such as the adoption of crouch posture, may impair their patterns of CoP oscillation and muscle recruitment in lower limbs. These factors might have led them to use a stability strategy of freezing their degrees of freedom in the ankle joint, using preferably the hip strategy for stability [5], which involves greater displacements in the ML axis [39]. Thus, these children may not present significant differences in AP Amp, but significant ones in ML Amp, as we found in our results.

No differences were found in postural oscillation between children GMFCS levels level I and II in none of the conditions. The main functional differences observed among level I and II are related with the limitations in independent walking (level I are able to perform it in internal and external environment, whereas level II are not). This specific feature (ability to walk independently) was not expected to interfere with the accomplishment of the tasks in this study, since children of both levels were able to accomplish the tasks without assistance. In addition, the motor impairments observed in level I and II of GMFCS are considered mild ones [40], with some studies even including these levels in the same group [2]. Anyway, these results should be interpreted with caution taking into account the small size of our sample.

The knowledge about the greater biomechanical demands involving quiet stance following body movements draws attention to professionals involved with rehabilitation about the importance of training balance not only in static situations, but also during tasks which movement the CoM, such as the STS movement. We believe that balance training in such dynamical conditions would improve postural control of children with $\mathrm{CP}[1,41]$, thus preparing them to functional tasks of daily life.

In addition, according to our results it is also important to point out that in balance assessments the preceding motor task influences the outcomes. Therefore, it seems to be highly important to standardize the preceding motor task prior to any balance measurement to increase the precision of the studies. Moreover, we suggest that a preceding STS will increase the sensitivity of the balance test, although further studies are warranted.

Our study evaluated postural control of children with CP during quiet stance considering classical descriptive parameters. Further studies should address this topic using dynamic posturography, in order to better comprehend the variability present in patterns of motor behavior [42].

\section{Conclusion}

Quiet stance following STS movement involves greater postural oscillation compared with quiet stance alone. Moreover, the structural impairments present in children with $\mathrm{CP}$ makes these children more susceptible to loss of stability when it is required to keep static standing posture following STS movement.

\section{Acknowledgment}

The authors declare that the study was financially supported by a grant from FAPESP (2013/13380-6).

\section{References}

1. Woollacott MH \& Shumway-Cook A. Postural dysfunction during standing and walking in children with cerebral palsy: What are the underlying problems and what the new therapies might improve balance? Neural Plasticity. 2005; 12: $211-219$

2. Mancini MC, Alves ACM, Schaper C, Figueiredo EM, Sampaio RF, Coelho ZA, et al. Gravidade da Paralisia Cerebral e Desempenho Funcional. Brazilian Journal of Physical Therapy. 2004; 8: 253-260.

3. Pavão SL, dos Santos AN, Woollacott MH, \& Rocha NACF. Assessment of postural control in children with cerebral palsy: A review. Research in Developmental Disabilities. 2013; 34: 1367-1375.

4. Duarte M, \& Freitas SMSF. Revision of posturography based on force plate for balance evaluation. Brazilian Journal of Physical Therapy. 2010; 14: 183192.

5. Nashner LM, Shumway-Cook A \& Marin O. Stance posture control in selective groups of children with cerebral palsy: Deficits in sensory organization and muscular coordination. Experimental Brain Research. 1983; 49: 393-409.

6. Austad H, van der Meer ALH. Prospective dynamic balance control in healthy children and adults. Experimental Brain Research. 2007; 181: 289-295. 
7. Huxham FE, Goldie PA, Patla AE. Theoretical considerations in balance assessment. Australian Journal of Physiotherapy. 2001; 47: 89-100.

8. Barela JA, Focks GMJ, Hilgeholt T, Barela AMF, Carvalho RP \& Savelsbergh GJP. Perception-action and adaptation in postural control of children and adolescents with cerebral palsy. Research in Developmental Disabilities. 2001; 32: 2075-2083.

9. Bigongiari A, Souza FA, Franciulli PM, Neto SER, Araujo RC \& Mochizuki L. Anticipatory and compensatory postural adjustments in sitting in children with cerebral palsy. Human Movement Science. 2001; 30: 648-657.

10. Girolami GL, Shiratori T \& Aruin AS. Anticipatory postural adjustments in children with hemiplegia and diplegia. Journal of Electromyography and Kinesiology. 2011; 6: 988-997.

11. Rha DW, Kim DJ \& Park ES. Effect of hinged ankle-foot orthoses on standing balance control in children with bilateral spastic cerebral palsy. Yonse Medical Journal. 2010; 52: 746-752.

12. Donker SF, Ladebt A, Roerdink M, Savelsbergh GJP \& Beek PJ. Children with cerebral palsy exhibit greater and more postural sway than typically developing children. Experimental Brain Research. 2008; 184: 363-370.

13. Rose J, Wolff DR, Jones VK, Bloch DA, Oehlert JW \& Gamble JG. Postura balance in children with cerebral palsy. Developmental Medicine and Child Neurology. 2002; 44: 58-63.

14. Reilly DS, Woollacott MH \& Donkelaar PV. The interaction between executive attention and postural control in dual task conditions: Children with cerebral palsy. Archives of Physical Medicine and Rehabilitation. 2008; 89: 834-842.

15. Wulf $\mathrm{G}, \mathrm{McNevin} \mathrm{M}$ \& Shea $\mathrm{CH}$. The automaticity of complex motor skill learning as a function of attentional focus. Quarterly Journal of Experimental Psychology. 2001; 54: 1143-1154.

16. Pavão SL, dos Santos AN, Oliveira $A B$ \& Rocha NACF. Postural control during sit-to-stand movement and its relationship with upright position in children with hemiplegic spastic cerebral palsy and typical developing Brazilian Journal of Physical Therapy. 2015; 19: 18-25.

17. Patla A, Frank J, Winter D. Assessment of balance control in the elderly: major issues. Phisiotherapy Canada. 1990; 42: 89-97.

18. Liao HF, Gan SM, Lee HJ \& Kim D. Effects of Weight Resistance on the Temporal Parameters and Electromyography of Sit-to-Stand Movements in Children with and Without Cerebral Palsy. American Journal of Physical Medicine and Rehabilitation. 2010; 89: 99-106.

19. Shumway-Cook A \& Woollacott MH. Motor control: Translating research into clinical practice. Fourth, North American Edition Edition. Chapter 3 Physiology of motor control. 2010; 46-82.

20. Yoshioka S, Nagano A, Hay DC \& Fukashiro S. Biomechanical analysis of the relation between movement time and joint moment development during a sit-to-stand task. BioMedical Engineering OnLine. 2009; 8: 1-9.

21. Medeiros DL, Conceicao JS, Graciosa MD, et al. The influence of seat heights and foot placement positions on postural control in children with cerebral palsy during a sit-to-stand task. Research in Developmental Disabilities. 2015; 43-44: 1-10.

22. Pavão SL, dos Santos AN, Oliveira AB, Rocha NACF. Functionality level and its relation to postural control during sitting-to-stand movement in children with cerebral palsy. Research in Developmental Disabilities. 2014; 35: 506511

23. Hsue BJ, Miller F \& Su FC. The dynamic balance of the children with cerebral palsy and typical developing during gait. Part I: Spatial relationship between COM and COP trajectories. Gait and Posture. 2009; 29: 465-470.

24. Liao HF, Jeng SF, Lai JS, Cheng CK \& Hu MH. The relation between standing balance and walking function in children with spastic diplegic cerebral palsy. Developmental Medicine and Child Neurology. 1997; 39: 106-112.
25. Buckley TA, Pitsikoulis C, Hass CJ. Dynamic postural stability during sit-towalk transitions in Parkinson disease patients. Movement Disorder. 2008; 23 : 1274-1280.

26. Dos Santos AN, Pavão SL, Santiago PRP, Salvini TF \& Rocha NACF. Sit-tostand movement in children with hemiplegic cerebral palsy: Relationship with knee extensor torque and social participation. Research in Developmental Disabilities. 2013; 34: 2023-2032.

27. Zaino CA \& McCoy SL. Reliability and comparison of electromyographic and kinetic measurements during a standing reach task in children with and without cerebral palsy. Gait and Posture. 2008; 27: 128-137.

28. Freitas SMSF \& Duarte M. Métodos de Análise do Controle Postural. São Paulo: Laboratório de Biofísica, Escola de Educação Física e Esporte, Universidade de São Paulo. 2006.

29. Kralj A, Jaeger RJ \& Munih M. Analysis of standing up and standing down in humans. Journal of Biomechanics. 1990; 23: 1123-1138.

30. Pavão SL, Silva FPS, Savelsbergh GJP, Rocha NACF. Use of Sensory Information During Postural Control in Children With Cerebral Palsy: Systematic Review. Journal of Motor Behavior. 2015; 47: 291-301.

31. Mourey F, Grishin A, dÁthis P, Pozzo T, Stapley P. Standing up from a chair as a dynamic equilibrium task: A comparison between young and elderly subjects. Journal of Gerontology. 2000; 55: 425-431.

32. Park ES, Park C, Lee HJ \& Kim D. The characteristics of sit-to-stand transfe in young children with cerebral palsy based on kinematic and kinetic data. Gait and Posture. 2003; 17: 43-49.

33. Cobb SC, Tis LL, Johnson BF \& Higbie EJ. The effect of forefoot varus on postural stability. Journal of Orthopaedic \& Sports Physical Therapy. 2004; 34: 79-85.

34. Johnson MB \& Van Emmerik REA. Is head-on-trunk extension a proprioceptive mediation of postural control and sit-to-stand characteristics? Journal of Motor Behavior. 2011; 43: 491-498.

35. Horak F, Shupert C. The role of the vestibular system in postural control. In Herdman S, ed. Vestibular Rehabilitation. New York: FA Davis. 1994; 22-46.

36. Papadelis C, Ahtam B, Nazarova M, Nimec D, Snyder B, Grant PE, et al. Cortical somatosensory reorganization in children with spastic cerebral palsy: a multimodal neuroimaging study. Frontiers in Human Neuroscience; 2014 8: 725

37. Bleyenheuft $Y \&$ Gordon AM. Precision grip control, sensory impairments and their interaction in children with hemiplegic cerebral palsy: A systematic review. Research in Developmental Disabilities. 2013; 34: 3014-3028.

38. Massion J. Postural control systems in developmental perspective Neuroscience and Biobehavioral Reviews. 1998; 22: 465-472.

39. Ferdjallah M, Harris GF, Smith P \& Wertsch JJ. Analysis of postural control synergies during quiet standing in health children and children with cerebral palsy. Clinical Biomechanics. 2002; 17: 203-210.

40. Saavedra S, Woollacott M \& van Donkelaar P. Head stability during quie sitting in children with cerebral palsy. Experimental Brain Research. 2010; 201: 213-223.

41. Pavão SL, Arnoni JLB, Oliveira AKC \& Rocha NACF. Impact of a virtua reality-based intervention on motor performance and balance of a child with cerebral palsy: A case study. Revista Paulista de Pediatria 2014; 32: 389394.

42. Harbourne R \& Stergiou N. Nonlinear analysis of the development of sitting postural control. Developmental Psychobiology. 2003; 42: 368-377.
Phys Med Rehabil Int - Volume 4 Issue 3 - 2017 ISSN : 2471-0377 | www.austinpublishinggroup.com Pavão et al. () All rights are reserved
Citation: Pavão SL, de Oliveira Sato T and Rocha NACF. Differences in Postural Oscillation during Quiet Stance Alone and Quiet Stance Following Sit-To-Stand Movement in Children with Cerebral Palsy. Phys Med Rehabil Int. 2017; 4(3): 1121. 